\title{
Some Presic Type Generalizations of the Banach Contraction Principle
}

\author{
K.P.R. RaO, Md. Mustaq Ali and Brian Fisher
}

Abstract. In this paper, we extend and generalize Presic Type theorems for a pair of maps and Jungck type maps.

\section{Introduction AND PRELIMINARIES}

In 1932 Banach [2] proved the following theorem:

Theorem $1.1([2])$. Let $(X, d)$ be a complete metric space and let $T$ be a mapping of $X$ into $X$ satisfying the inequality $d(T x, T y) \leq \lambda d(x, y)$ for all $x, y \in X$, where $0 \leq \lambda<1$. Then $T$ has a unique fixed point in $X$.

Since then, many generalizations of this principle have been made by several authors. Considering the convergence of certain sequences Presic [3] proved the following theorem.

Theorem $1.2([3])$. Let $(X, d)$ be a complete metric space, $k$ a positive integer and let $T$ be a mapping of $X^{k}$ into $X$, satisfying the following contractive type condition

$$
\begin{aligned}
& d\left(T\left(x_{1}, x_{2}, x_{3}, \ldots, x_{k}\right), T\left(x_{2}, x_{3}, x_{4}, \ldots, x_{k}, x_{k+1}\right)\right) \\
\leq & q_{1} d\left(x_{1}, x_{2}\right)+q_{2} d\left(x_{2}, x_{3}\right)+\cdots+q_{k} d\left(x_{k}, x_{k+1}\right)
\end{aligned}
$$

for every $x_{1}, x_{2}, x_{3}, x_{4}, \ldots, x_{k}, x_{k+1} \in X$, where $q_{1}, q_{2}, \ldots, q_{k}$ are non-negative constants such that $q_{1}+q_{2}+\ldots+q_{k}<1$. Then there exists a unique point $x \in X$ such that $T(x, x, x, \ldots, x)=x$.

Moreover, if $x_{1}, x_{2}, x_{3}, \ldots, x_{k}$ are arbitrary points in $X$ and if for all $n \in$ $N, x_{n+k}=T\left(x_{n}, x_{n+1}, \ldots, x_{n+k-1}\right)$, then the sequence $\left\{x_{n}\right\}$ is convergent and $\lim x_{n}=T\left(\lim x_{n}, \lim x_{n}, \ldots, \lim x_{n}\right)$.

Ciric and Presic [1] generalized Theorem 1.2 as follows:

Received by the editors 18.10 .2010 .

2000 Mathematics Subject Classification. Primary: 47H10; 54H25.

Key words and phrases. Weakly $k$-compatible maps, Jungck Type maps, Common fixed point. 
Theorem 1.3. Let $(X, d)$ be a complete metric space, $k$ a positive integer and let $T$ be a mapping of $X^{k}$ into $X$ satisfying the following contractive type condition

$$
\begin{aligned}
& d\left(T\left(x_{1}, x_{2}, x_{3}, \ldots, x_{k}\right), T\left(x_{2}, x_{3}, x_{4}, \ldots, x_{k}, x_{k+1}\right)\right) \\
\leq & \lambda \max \left\{d\left(x_{i}, x_{i+1}\right) / 1 \leq i \leq k\right\}
\end{aligned}
$$

for every $x_{1}, x_{2}, x_{3}, x_{4}, \ldots, x_{k}, x_{k+1} \in X$, where $0<\lambda<1$. Then there exists a point $x \in X$ such that $T(x, x, x, \ldots, x)=x$.

Moreover, if $x_{1}, x_{2}, x_{3}, \ldots, x_{k}$ are arbitrary points in $X$ and if for all $n \in$ $N, x_{n+k}=T\left(x_{n}, x_{n+1}, \ldots, x_{n+k-1}\right)$, then the sequence $\left\{x_{n}\right\}$ is convergent and $\lim x_{n}=T\left(\lim x_{n}, \lim x_{n}, \ldots, \lim x_{n}\right)$. If in addition, we suppose that on the diagonal $\Delta \subset X^{k}$, the condition

$$
d(T(u, u, \ldots, u), T(v, v, \ldots, v))<d(u, v)
$$

holds for all distinct $u, v \in X$, then $x$ is the unique point in $X$ with $T(x, x, \ldots, x)=x$.

Now in this paper we extend and generalize the above theorems for a pair of mappings and Jungck type mappings.

\section{MAIN RESUlT}

Theorem 2.1. Let $(X, d)$ be a complete metric space, $k$ a positive integer and let $S, T$ be mappings of $X^{2 k}$ into $X$ satisfying the following contractive type conditions

$$
\begin{aligned}
& d\left(S\left(x_{1}, x_{2}, \ldots, x_{2 k-1}, x_{2 k}\right), T\left(x_{2}, x_{3}, \ldots, x_{2 k}, x_{2 k+1}\right)\right) \\
\leq & \lambda \max \left\{d\left(x_{i}, x_{i+1}\right): 1 \leq i \leq 2 k\right\},
\end{aligned}
$$

for all $x_{1}, x_{2}, \ldots, x_{2 k}, x_{2 k+1} \in X$ and

$$
\begin{aligned}
& d\left(T\left(y_{1}, y_{2}, \ldots, y_{2 k-1}, y_{2 k}\right), S\left(y_{2}, y_{3}, \ldots, y_{2 k}, y_{2 k+1}\right)\right) \\
\leq & \lambda \max \left\{d\left(y_{i}, y_{i+1}\right): 1 \leq i \leq 2 k\right\},
\end{aligned}
$$

for all $y_{1}, y_{2}, \ldots, y_{2 k}, y_{2 k+1} \in X$, where $0 \leq \lambda<1$.

Suppose $x_{1}, x_{2}, \ldots, x_{2 k}$ are arbitrary points in $X$ and for all $n \in N$ let

$$
x_{2 k+2 n-1}=S\left(x_{2 n-1}, x_{2 n}, x_{2 n+1}, \ldots, x_{2 n+2 k-2}\right)
$$

and

$$
x_{2 k+2 n}=T\left(x_{2 n}, x_{2 n+1}, x_{2 n+2}, \ldots, x_{2 n+2 k-1}\right) .
$$

Then the sequence $\left\{x_{n}\right\}$ is convergent to some $x \in X$ such that

$$
S(x, x, \ldots, x)=x=T(x, x, \ldots, x) .
$$

In addition, if

(i) $2 k \lambda<1$, or

(ii) $d(S(u, u, \ldots, u), T(v, v, \ldots, v))<d(u, v)$,

for all distinct $u, v \in X$, then $x$ is the unique point satisfying (A). 
Proof. Let $\alpha_{n}=d\left(x_{n}, x_{n+1}\right)$. We claim that $\alpha_{n} \leq K \theta^{n}$, for all $n \in N$, where $\theta=\lambda^{1 / 2 k}$ and $K=\max \left\{\alpha_{1} / \theta^{1}, \alpha_{2} / \theta^{2}, \ldots, \alpha_{2 k} / \theta^{2 k}\right\}$. By selection of $K$ we have $\alpha_{n} \leq K \theta^{n}$ for $n=1,2, \ldots, 2 k$.

Now

$$
\begin{aligned}
& \alpha_{2 k+1}=d\left(x_{2 k+1}, x_{2 k+2}\right) \\
& =d\left(S\left(x_{1}, x_{2}, \ldots, x_{2 k-1}, x_{2 k}\right), T\left(x_{2}, x_{3}, \ldots, x_{2 k}, x_{2 k+1}\right)\right) \\
& \left.\leq \lambda \max \left\{d\left(x_{i}, x_{i+1}\right): i=1,2, \ldots, 2 k\right\} \quad \text { (by }(2.1)\right) \\
& =\lambda \max \left\{\alpha_{1}, \alpha_{2}, \ldots, \alpha_{2 k-1}, \alpha_{2 k}\right\} \\
& \leq \lambda \max \left\{K \theta, K \theta^{2}, \ldots, K \theta^{2 k-1}, K \theta^{2 k}\right\} \\
& =\lambda K \theta \\
& =K \theta^{2 k+1} \quad\left(\text { since } \theta=\lambda^{1 / 2 k}\right)
\end{aligned}
$$

and so $\alpha_{2 k+1} \leq K \theta^{2 k+1}$.

Similarly

$$
\begin{aligned}
& \alpha_{2 k+2}=d\left(x_{2 k+2}, x_{2 k+3}\right) \\
& =d\left(T\left(x_{2}, x_{3}, \ldots, x_{2 k}, x_{2 k+1}\right), S\left(x_{3}, x_{4}, \ldots, x_{2 k+1}, x_{2 k+2}\right)\right) \\
& \left.\leq \lambda \max \left\{d\left(x_{i}, x_{i+1}\right): i=2,3, \ldots, 2 k+1\right\} \quad \text { (by }(2.2)\right) \\
& =\lambda \max \left\{\alpha_{i} / i=2,3, \ldots, 2 k+1\right\} \\
& \leq \lambda \max \left\{K \theta^{2}, K \theta^{3}, \ldots, K \theta^{2 k+1}\right\} \\
& =\lambda K \theta^{2} \\
& =K \theta^{2 k+2} \quad\left(\text { since } \theta=\lambda^{1 / 2 k}\right)
\end{aligned}
$$

and so $\alpha_{2 k+2} \leq K \theta^{2 k+2}$. Hence our claim is true.

Now, by our claim, for any $n, p \in N$, we have

$$
\begin{aligned}
& d\left(x_{n}, x_{n+p}\right) \leq d\left(x_{n}, x_{n+1}\right)+d\left(x_{n+1}, x_{n+2}\right)+\ldots+d\left(x_{n+p-1}, x_{n+p}\right) \\
= & \alpha_{n}+\alpha_{n+1}+\ldots+\alpha_{n+p-1} \\
\leq & K \theta^{n}+K \theta^{n+1}+\ldots+K \theta^{n+p-1} \\
\leq & K\left(\theta^{n}+\theta^{n+1}+\ldots+\theta^{n+p-1}+\ldots\right) \\
= & K \frac{\theta^{n}}{1-\theta} \rightarrow 0 \quad(\text { as } n \rightarrow \infty) .
\end{aligned}
$$

Hence $\left\{x_{n}\right\}$ is a Cauchy sequence. Since $X$ is a complete metric space, there exists a point $x \in X$ such that $x=\lim _{n \rightarrow \infty} x_{n}$. Then for any integer 
$n$, using (2.1) and (2.2), we have

$$
\begin{aligned}
d( & \left.S(x, x, \ldots, x), x_{2 n+2 k-1}\right)=d\left(S(x, x, \ldots, x), S\left(x_{2 n-1}, x_{2 n}, \ldots, x_{2 n+2 k-2}\right)\right) \\
\leq d & \left.S(x, x, \ldots, x), T\left(x, x, \ldots, x, x_{2 n-1}\right)\right) \\
& +d\left(T\left(x, x, \ldots, x, x_{2 n-1}\right), S\left(x, x, \ldots, x_{2 n-1}, x_{2 n}\right)\right) \\
& +d\left(S\left(x, x, \ldots, x, x_{2 n-1}, x_{2 n}\right), T\left(x, x, \ldots, x, x_{2 n-1}, x_{2 n}, x_{2 n+1}\right)\right) \\
& +d\left(T\left(x, x, \ldots, x, x_{2 n}, x_{2 n+1}\right), S\left(x, x, \ldots, x, x_{2 n}, x_{2 n+1}, x_{2 n+2}\right)\right)+\ldots \\
& +d\left(S\left(x, x, x_{2 n-1}, x_{2 n}, \ldots, x_{2 n+2 k-4}\right), T\left(x, x_{2 n-1}, x_{2 n}, \ldots, x_{2 n+2 k-3}\right)\right) \\
& +d\left(T\left(x, x_{2 n-1}, x_{2 n}, \ldots, x_{2 n+2 k-3}\right), S\left(x_{2 n-1}, x_{2 n}, \ldots, x_{2 n+2 k-2}\right)\right) \\
\leq \lambda & \left.d x, x_{2 n-1}\right)+\lambda \max \left\{d\left(x, x_{2 n-1}\right), d\left(x_{2 n-1}, x_{2 n}\right)\right\} \\
& +\lambda \max \left\{d\left(x, x_{2 n-1}\right), d\left(x_{2 n-1}, x_{2 n}\right), d\left(x_{2 n}, x_{2 n+1}\right)\right\}+\ldots \\
& +\lambda \max \left\{d\left(x, x_{2 n-1}\right), d\left(x_{2 n-1}, x_{2 n}\right), d\left(x_{2 n}, x_{2 n+1}\right), d\left(x_{2 n+1}, x_{2 n+2}\right)\right\} \\
& +\ldots+\lambda \max \left\{d\left(x, x_{2 n-1}\right), d\left(x_{2 n-1}, x_{2 n}\right), \ldots, d\left(x_{2 n+2 k-4}, x_{2 n+2 k-3}\right)\right\} \\
& +\lambda \max \left\{d\left(x, x_{2 n-1}\right), d\left(x_{2 n-1}, x_{2 n}\right), \ldots, d\left(x_{2 n+2 k-3}, x_{2 n+2 k-2}\right)\right\} .
\end{aligned}
$$

Taking the limit as $n \rightarrow \infty$, we get

$$
d(S(x, x, \ldots, x), x) \leq 0
$$

and so $S(x, x, \ldots, x)=x$.

From (2.1), we have

$$
d(x, T(x, x, \ldots, x))=d(S(x, x, \ldots, x), T(x, x, \ldots, x))=0
$$

and so $T(x, x, \ldots, x)=x$.

To prove the uniqueness of $x$, we suppose that there exists a point $y \neq x$ in $X$ such that

$$
S(y, y, \ldots, y)=y=T(y, y, \ldots, y) .
$$

Suppose (i) holds so that $2 k \lambda<1$.

$$
\begin{aligned}
& d(x, y)=d(S(x, x, \ldots, x), T(y, y, \ldots, y)) \\
& \leq d(S(x, x, \ldots, x), T(x, x, \ldots, x, y))+d(T(x, x, \ldots, x, y), S(x, x, \ldots, x, y, y)) \\
& \quad+d(S(x, x, \ldots, x, y, y), T(x, x, \ldots, x, y, y, y)) \\
& \quad+d(T(x, x, \ldots, x, y, y, y), S(x, x, \ldots, x, y, y, y, y)) \\
& \quad+\ldots+d(S(x, x, y, y, \ldots, y), T(x, y, y, \ldots, y)) \\
& \quad+d(T(x, y, y, \ldots, y), S(y, y, y, \ldots, y)) \\
& \quad+d(S(y, y, y, \ldots, y), T(y, y, y, \ldots, y)) \\
& \leq \lambda d(x, y)+\lambda d(x, y)+\lambda d(x, y)+\ldots+\lambda d(x, y)+\lambda d(x, y)+0 \\
& =2 K \lambda d(x, y)<d(x, y),
\end{aligned}
$$

a contradiction. Therefore $y=x$. 
Suppose (ii) holds. Then

$$
d(x, y)=d(S(x, x, \ldots, x), T(y, y, \ldots, y))<d(x, y),
$$

a contradiction and again $y=x$.

Corollary 2.1. Let $(X, d)$ be a complete metric space, $k$ a positive integer and let $S, T$ be mappings of $X^{2 k}$ into $X$ satisfying

$$
\begin{aligned}
& d\left(S\left(x_{1}, x_{2}, \ldots, x_{2 k-1}, x_{2 k}\right), T\left(x_{2}, x_{3}, \ldots, x_{2 k}, x_{2 k+1}\right)\right) \\
\leq & q_{1} d\left(x_{1}, x_{2}\right)+q_{2} d\left(x_{2}, x_{3}\right)+\ldots+q_{2 k} d\left(x_{2 k}, x_{2 k+1}\right),
\end{aligned}
$$

for all $x_{1}, x_{2}, x_{3}, \ldots, x_{2 k}, x_{2 k+1} \in X$ and

$$
\begin{aligned}
& d\left(T\left(y_{1}, y_{2}, \ldots, y_{2 k-1}, y_{2 k}\right), S\left(y_{2}, y_{3}, \ldots, y_{2 k}, y_{2 k+1}\right)\right) \\
\leq & q_{1} d\left(y_{1}, y_{2}\right)+q_{2} d\left(y_{2}, y_{3}\right)+\ldots+q_{2 k} d\left(y_{2 k}, y_{2 k+1}\right)
\end{aligned}
$$

for all $y_{1}, y_{2}, \ldots, y_{2 k}, y_{2 k+1} \in X$, where $q_{1}, q_{2}, \ldots, q_{2 k}$ are non-negative constants such that $q_{1}+q_{2}+\ldots+q_{2 k}<1$. Then there exists unique $x \in X$ such that

$$
S(x, x, x, \ldots, x)=x=T(x, x, x, \ldots, x) .
$$

Proof. (2.3) and (2.4) imply the conditions (2.1) and (2.2) respectively with $\lambda=q_{1}+q_{2}+\ldots+q_{2 k}$. Now from Theorem 2.1, there exists $x \in X$ such that

$$
S(x, x, \ldots, x)=x=T(x, x, \ldots, x) .
$$

To prove the uniqueness of $x$, suppose there exists a point $y \neq x$ in $X$ such that

Then

$$
S(y, y, \ldots, y)=y=T(y, y, \ldots, y) .
$$

$$
\begin{aligned}
& d(x, y)=d(S(x, x, \ldots, x), T(y, y, \ldots, y)) \\
& \leq d(S(x, x, \ldots, x), T(x, x, \ldots, x, y))+d(T(x, x, \ldots, x, y), S(x, x, \ldots, x, y, y)) \\
& \quad+d(S(x, x, \ldots, x, y, y), T(x, x, \ldots, x, y, y, y)) \\
& \quad+d(T(x, x, \ldots, x, y, y, y), S(x, x, \ldots, x, y, y, y, y))+\ldots \\
& \quad+d(S(x, x, y, y, \ldots, y), T(x, y, y, \ldots, y)) \\
& \quad+d(T(x, y, y, \ldots, y), S(y, y, y, \ldots, y)) \\
& \quad+d(S(y, y, y, \ldots, y), T(y, y, y, \ldots, y)) \\
& \leq q_{2 k} d(x, y)+q_{2 k-1} d(x, y)+\ldots+q_{2} d(x, y)+q_{1} d(x, y)+0 \\
& =\left(q_{1}+q_{2}+\ldots+q_{2 k-1}+q_{2 k}\right) d(x, y)<d(x, y),
\end{aligned}
$$

which is a contradiction. Therefore $y=x$.

Definition 2.1. Let $X$ be a non empty set, let $T$ be a mapping of $X^{k}$ into $X$ and let $f$ be a mapping of $X$ into $X$. Then $(f, T)$ is said to be weakly a $k$-compatible pair if $f(T(p, p, \ldots, p))=T(f p, f p, \ldots, f p)$, whenever $p \in X$ is such that $f p=T(p, p, \ldots, p)$. 
Theorem 2.2. Let $(X, d)$ be a metric space, $k$ a positive integer, let $T$ be a mapping of $X^{k}$ into $X$ and let $f$ be a mapping of $X$ into $X$ satisfying

$$
\begin{aligned}
& d\left(T\left(x_{1}, x_{2}, x_{3}, \ldots, x_{k}\right), T\left(x_{2}, x_{3}, x_{4}, \ldots, x_{k}, x_{k+1}\right)\right) \\
\leq & \lambda \max \left\{d\left(f x_{i}, f x_{i+1}\right): 1 \leq i \leq k\right\},
\end{aligned}
$$

for all $x_{1}, x_{2}, x_{3}, x_{4}, \ldots, x_{k}, x_{k+1} \in X$, where $0<\lambda<1$ and

$$
d(T(u, u, \ldots, u), T(v, v, \ldots, v))<d(f u, f v),
$$

for all distinct $u, v \in X$. Suppose further that $T\left(X^{k}\right) \subseteq f(X), f(X)$ is complete and $(f, T)$ is a weakly $k$-compatible pair. Then there exists a unique point $z \in X$ such that

$$
f z=z=T(z, z, \ldots, z) .
$$

Proof. Let $x_{1}, x_{2}, \ldots, x_{k}$ be arbitrary points in $X$ and define

$$
f x_{n+k}=T\left(x_{n}, x_{n+1}, \ldots, x_{n+k-1}\right)
$$

for all $n \in N$. By proceeding as in [1], we can prove that $\left\{f x_{n}\right\}$ is a Cauchy sequence in $f(X)$. Since $f(X)$ is complete, there exists a point $z \in f(X)$ such that $f x_{n} \longrightarrow z$. Hence there exists a point $p \in X$ such that $z=f p$.

Now consider

$$
\begin{aligned}
d( & \left.f x_{n+k}, T(p, p, \ldots, p)\right)=d\left(T(p, p, \ldots, p), T\left(x_{n}, x_{n+1}, \ldots, x_{n+k-1}\right)\right) \\
\leq d & \left(T(p, p, \ldots, p), T\left(p, p, \ldots, p, x_{n}\right)\right) \\
\quad & +d\left(T\left(p, p, \ldots, p, x_{n}\right), T\left(p, p, \ldots, p, x_{n}, x_{n+1}\right)\right) \\
\quad & +d\left(T\left(p, p, \ldots, p, x_{n}, x_{n+1}\right), T\left(p, p, \ldots, p, x_{n}, x_{n+1}, x_{n+2}\right)\right) \\
\quad & +d\left(T\left(p, p, \ldots, p, x_{n}, x_{n+1}, x_{n+2}\right), T\left(p, p, \ldots, p, x_{n}, x_{n+1}, x_{n+2}, x_{n+3}\right)\right) \\
& +\ldots+d\left(T\left(p, x_{n}, x_{n+1}, \ldots, x_{n+k-2}\right), T\left(x_{n}, x_{n+1}, \ldots, x_{n+k-1}\right)\right) \\
\leq & d\left(f p, f x_{n}\right)+\lambda \max \left\{d\left(f p, f x_{n}\right), d\left(f x_{n}, f x_{n+1}\right)\right\} \\
\quad & +\lambda \max \left\{d\left(f p, f x_{n}\right), d\left(f x_{n}, f x_{n+1}\right), d\left(f x_{n+1}, f x_{n+2}\right)\right\} \\
& +\lambda \max \left\{d\left(f p, f x_{n}\right), d\left(f x_{n}, f x_{n+1}\right), d\left(f x_{n+1}, f x_{n+2}\right), d\left(f x_{n+2}, f x_{n+3}\right)\right\} \\
& +\ldots+\lambda \max \left\{d\left(f p, f x_{n}\right), d\left(f x_{n}, f x_{n+1}\right), \ldots, d\left(f x_{n+k-2}, f x_{n+k-1}\right)\right\} .
\end{aligned}
$$

Letting $n \longrightarrow \infty$ we get

$$
d(f p, T(p, p, \ldots, p)) \leq 0
$$

so that $f p=T(p, p, \ldots, p)$.

Since $(f, T)$ is weakly $k$-compatible we have

$$
f(T(p, p, \ldots, p))=T(f p, f p, \ldots, f p)
$$

and so

Thus

$$
f^{2} p=f(f p)=f(T(p, p, \ldots, p))=T(f p, f p, \ldots, f p) .
$$

$$
f z=T(z, z, \ldots, z) .
$$


We now have

$$
d\left(f^{2} p, f p\right)=d(T(f p, f p, \ldots, f p), T(p, p, \ldots, p))<d\left(f^{2} p, f p\right),
$$

which is a contradiction. Therefore $f^{2} p=f p$ so that $f z=z$.

From (i), we now have

$$
z=f z=T(z, z, \ldots, z) .
$$

To prove uniqueness, suppose that there exists a point $z^{1} \neq z$ in $X$ such that

$$
z^{1}=f z^{1}=T\left(z^{1}, z^{1}, \ldots, z^{1}\right) .
$$

Then

$$
\begin{aligned}
d\left(z, z^{1}\right) & =d\left(T(z, z, \ldots, z),\left(T\left(z^{1}, z^{1}, \ldots, z^{1}\right)\right)\right. \\
& <d\left(f z, f z^{1}\right) \quad \text { from }(2.6) \\
& =d\left(z, z^{1}\right),
\end{aligned}
$$

which is a contradiction. Therefore $z=z^{1}$ proving that $z$ is the unique point satisfying (ii).

\section{REFERENCES}

[1] Lj.B. Ciric and S.B. Presic, On Presic type generalization of the Banach contraction mapping principle, Acta Math. Univ. Comenian, 76(2)(2007), 143-147.

[2] S. Banach, Theorie des operations lineaires, Manograic Mathematic Zne, Warsaw, 1932.

[3] S.B. Presic, Sur une classe d'inequations aux differences finies et sur la convergence de certaines suites, Publ. Inst. Math. (Beograd), 5(19)(1965), 75-78.

K.P.R. Rao and Md. Mustaq Ali Department of Applied Mathematics Dr. M.R. Appa Row Campus Acharya Nagarjuna University NUZVID - 521201, Krishna Dt., A.P. INDIA

E-mail address: kprrao2004@yahoo.com

\section{BRIAN Fisher}

Department of Mathematics

UNIVERSITY OF LEICESTER

LEICESTER, LE1 7RH

U.K.

E-mail address: fbr@leicester.ac.uk 
